MATEC Web of Conferences 37, 01002 (2015)

DOI: $10.1051 /$ matecconf $/ 20153701002$

(C) Owned by the authors, published by EDP Sciences, 2015

\title{
EXPERIMENTAL RESEARCH OF MUTUAL INFLUENCE OF SEVERAL CONSECUTIVE WATER DROPLETS ON REGULARITIES OF THEIR EVAPORATION THROUGH HIGH- TEMPERATURE
}

\author{
Dmitrii V. Antonov ${ }^{1}$, Roman S. Volkov ${ }^{1}$, Pavel A. Stizhak ${ }^{1, a}$ \\ ${ }^{1}$ National Research Tomsk Polytechnic University, 634050 Tomsk, Russia
}

\begin{abstract}
Experimental research of the main integral characteristic of several water droplets at their evaporation through high-temperature gases $(1100 \mathrm{~K})$ was carried out. Features of water droplet movement in a high-temperature gas stream are established. Characteristics of droplet movement at a variety of their initial parameters in a wide range was established by high-speed video registration preservers «Phantom» and software packages «Tema Automotive» and «Phantom Camera Control». The main integral characteristics of several water droplets in the different distance between them were installed.
\end{abstract}

\section{Introduction}

The most perspective question in a modern world is a question which connects with research of the movement process of consecutive water droplets through high-temperature gases. These questions are actual for different spheres such as thermal or flame treatment of water, emulsions and suspensions [1, 2] produced on its base. The main drawback of modern systems of thermal cleaning liquids is high fuel consumption for the implementation of the evaporation of water droplets and the necessity for droplet re-transmission through the heating chamber due to the significant liquid entrainment by gases, subsidence and condensation on the walls of its chambers. Entrainment of droplets by combustion products is an important issue for modern fire extinguishing systems by atomized streams $[2,3,4]$. Until recently, all this is due to the lack of approaches and methods for the reliable experimental studies of complex of interrelated occurring heat and mass transfer processes, phase transformations and chemical response for gas-vapor-droplet systems.

The aim of this work is an experimental research of the mutual influence of several moving water droplets on the laws of their evaporation during motion in a stream of high-temperature gases.

\footnotetext{
${ }^{a}$ Corresponding author: pavelspa@tpu.ru
} 


\section{Experimental setup and procedure}

The experimental setup was used at experimentations, in its main characteristic it is similar to that one used before $[2,3]$. This setup was used for registration of consecutive water droplet movement and for research of processes of evaporation and couple. The scheme of the experimental setup is shown in Fig. 1.

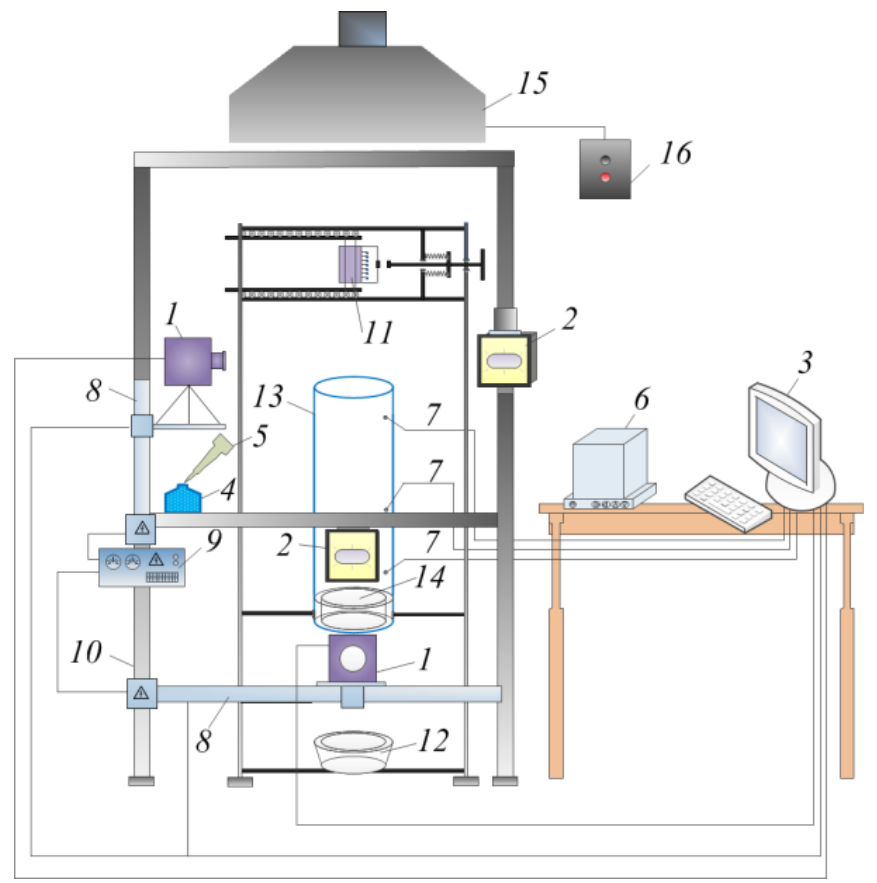

Figure 1. Scheme of experimental setup: 1 - high-speed digital camera; 2 - spotlight; 3 - personal computer; 4 vessel with experimental liquid; 5 - dosemeter; 6 - microbalance; 7 - thermocouples; 8 - motorized coordinate devices (MCD); 9 - power supply for MCD; 10 - mount; 11 - device of consecutive supply of drops; 12 droplet catcher; 13 - quartz glass cylinder; 14 - hollow cylinder with combustible liquid; 15 - exhaust ventilation; 16 - switching on and off board of exhaust ventilation

The stand was a registration system on the basis of high-speed cameras «Phantom V411» and «Phantom Miro M310» (frame frequency is up to $6 \cdot 10^{5}$ frames per second). For a generation of successive water droplets identical to each other, we used a device of consistent droplet supply specially designed for this purpose. This allows getting the initial values of the droplet motion velocity, which is about $0.2 \mathrm{~m} / \mathrm{s}$. Sizes (radii) of the generated droplets were in the range of 1-2 mm. To form the high temperature gas medium, it was used the vertical cylindrical channel from a quartz glass and hollow cylinder with a combustible liquid in internal medium. The space between the walls was filled with liquid fuels - kerosene, which was ignited later. The combustion product temperature $T_{\mathrm{g}}$ was about $1100 \mathrm{~K}$.

The initial water temperature $T_{\mathrm{w}}$ in the vessel varied between $275-350 \mathrm{~K}$ by setup and controlled by a chromel-copel (range of temperature measurement $-273-473 \mathrm{~K}$, the systematic error $\pm 1.5 \mathrm{~K}$ ) thermocouple. The maximum random error of measurement $T_{\mathrm{w}}$ was $3 \mathrm{~K}$.

Combustion product speed $U_{\mathrm{g}}$ in the cylinder was monitored using an injection system, measured by vane type anemometer «UnionTest AN110» (maximum error is $-0.1 \mathrm{~m} / \mathrm{s}$ ) and was about $1.5 \mathrm{~m} / \mathrm{s}$.

\section{Results and discussion}

In a process of this research, evaporation features of consecutive water droplets through hightemperature gases were established. A series of experiments on the registration of moving three water 
droplets in the flame of gasoline was conducted. The dependence of the initial size of the water droplets on changing the droplet size for three consecutive water droplets through high-temperature gases at various initial distances between them were obtained (Fig. 2a and Fig. 2b).

Furthermore, it was found that at the initial distances $L_{\mathrm{d}}$ between the first and second droplets of less than $10 \mathrm{~mm}$ after passing through a channel with high-temperature gases $1 \mathrm{~m}$ in length, the droplets were merged steadily (i.e., at the output from the high-temperature area $L=0$ ). With the growth of the initial distances between droplets, the impact of the first one on the moving conditions of the second one is weakened, and the distances $L$ grow nonlinearly.

The experiments showed that under considered conditions of heat exchange, the limiting initial distance, at which the influence of the first droplet on the movement conditions of the second one is very little, is $L_{\mathrm{d}}=35 \mathrm{~mm}$.

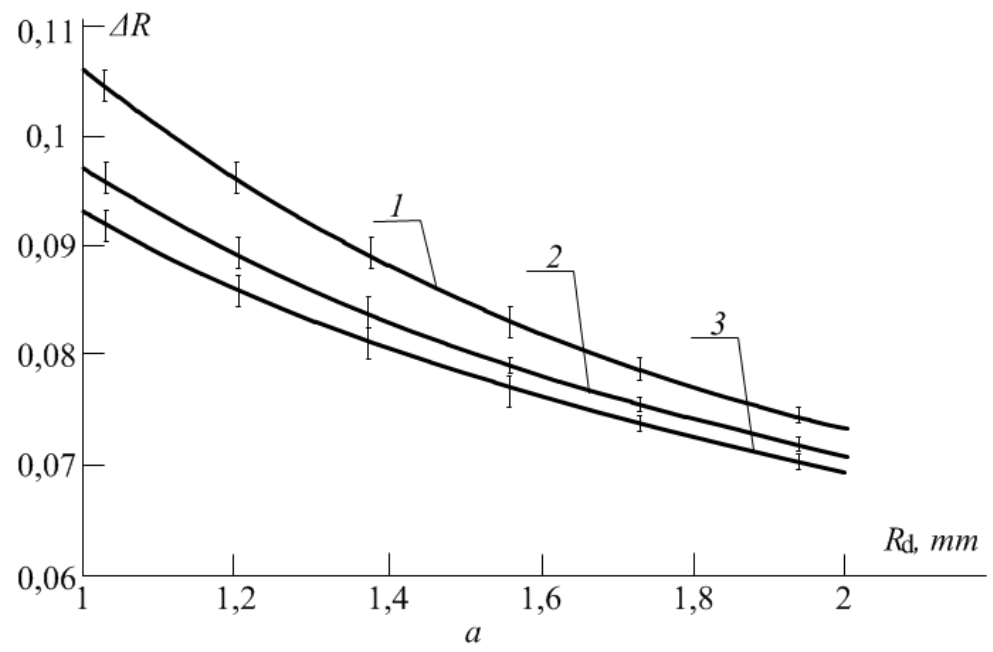

Figure 2a. The $\Delta R$ parameter for the first (1), second (2) and third (3) sequentially moving droplets after passing a high-temperature gaseous medium distance of $1 \mathrm{~m}$ in length at various initial distances between the droplets when $L_{\mathrm{d}}=8 \mathrm{~mm}$

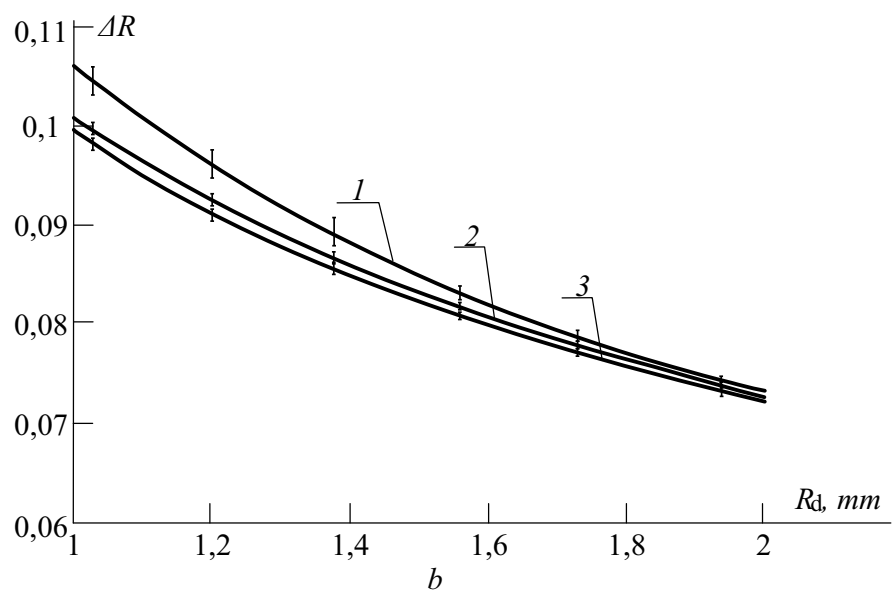

Figure $2 \mathbf{b}$. The $\Delta R$ parameter for the first (1), second (2) and third (3) sequentially moving droplets after passing a high-temperature gaseous medium distance of $1 \mathrm{~m}$ in length at various initial distances between the droplets when $L_{\mathrm{d}}=12 \mathrm{~mm}$ 


\section{MATEC Web of Conferences}

The obtained results illustrate the essentially nonlinear dependences for the selected characteristic droplet sizes. Fig. 1 shows the contrast of $\Delta \mathrm{R}$ for the first and subsequent droplets. It can be seen that after passing through the high temperature gas medium, the characteristic dimensions of the second and third droplets are very close. From this, it follows that each subsequent droplet of water undergoes evaporation speed reduction compared with the first one. This is due to a decrease in the surface temperature of the next water droplets (respectively, due to a decrease in temperature of gases around them).

\section{Conclusion}

The performed experimental researches have shown that the conditions of heating and evaporation of subsequent droplets differ as compared to the first one. The scale is determined by the differences between the initial distances between the droplets. The developed technique and established effects can be used to solve a large group of gas-vapor-droplet fundamental problems, as well as to carry out the prognostic assessment of evaporation completeness and droplet removal in the systems of thermal water purification and polydisperse fire-fighting.

This work was supported by the grant of the Russian Science Foundation (project No. 14-39-00003).

\section{References}

1. S. K. Aggarwal, F. Peng, J., Eng. for Gas Turbines and Power. 117, 453 (1995).

2. Yu. Varaksin, High Temperature 51, 377 (2013).

3. C.M. Augusto, J.B. Ribeiro, A.R. Gaspar, J.J. Costa, Int. J. Therm. Sci. 77, 19 (2014).

4. Z. H. Khan, Int. J. Heat and Mass Transf. 70, 610 (2014).

5. del Pino, L. Parras, M. Felli and R. Fernandez-Feria, Phys. Fluids. 23, 265 (2011).

6. Strizhak P.A., J. Eng. Phys. Thermophys. 86, 895 (2013). 